\title{
GRANULAR CELL TUMOR SIMULATING BREAST CANCER ON SCREENING MAMMOGRAM: CASE REPORT
}

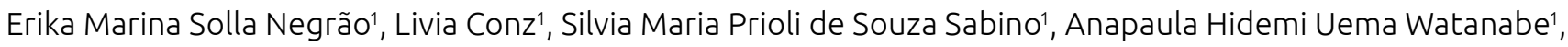
Jane Camargo da Silva Santos Picone', Edmundo Carvalho Mauad¹

${ }^{1}$ Barretos Cancer Hospital - Barretos (SP), Brazil.

Introduction: Granular cell tumor involving the breast parenchyma is very rare, representing between 5 and $15 \%$ of the presentations of this tumor. Due to the appearance of the image, it is confused with breast carcinoma; therefore, it can be a diagnostic challenge for medical mastologists, radiologists, and pathologists. Presentation of the case: We report the case of a 45-year-old woman who presented a lesion identified by ultrasound image with characteristics classified as highly suspected of malignancy (BIRADS 4c). The screening mammography detected a dense image of obscured margins, and the ultrasonography revealed a homogeneous irregular nodule with indistinct margins, located in the upper lateral quadrant of the right, posterior, and peripheral breast measuring $1.2 \mathrm{~cm}$. The lesion was subjected to percutaneous biopsy, and the histological examination combined with an immunohistochemical study revealed that it was a granular cell tumor. Conclusion: Although the granular cell tumor of the breast is a rare breast cancer, it must be considered in the differential diagnosis of lesions detected in imaging examinations. The granular cell tumor of the breast is a benign lesion, but the radiological findings suggest a malignant tumor, clinically and radiographically impossible to establish a definitive diagnosis without a biopsy.

Keywords: Granular; Cell Tumor; Breast Neoplasm; Breast Tumors. 